\title{
Answers to Dermatology Quiz
}

1. $\quad 1.1$ Tuberculoid leprosy / borderline tuberculoid leprosy with type 1 Lepra reaction

1.2 MDT-PB (Multidrug therapy-Paucibacillary) for 6 months and oral prednisolone

Type I lepra or reversal reaction are caused by an increased cell-mediated immune response to the leprosy bacillus or its remnants. Type I reaction is more common in patients between the two poles of the leprosy spectrum with immunologically unstable borderline forms, with approximately $30 \%$ being at risk. Triggers include poor nutrition, genetic defects in the cell-mediated immune response or cytokine production, stress, initiation of MDT therapy, hormonal imbalance, high bacillary load and high antibody titers to $M$. leprae antigens. Reactions can occur prior to initiation of MDT, during MDT and many years after completing MDT. Therefore, the direct cause of reactions is not whether the bacilli are alive or dead, but rather the changing status of the immune response in each individual at a particular time point.

2. 2.1 Subacute cutaneous lupus erythematosus

2.2 Topical steroids, avoiding exposure to sunlight with sunscreens and hydroxychloroquine

Subacute cutaneous lupus erythematosus (SCLE) is a non-scarring, photosensitive dermatosis that commonly develops on sun-exposed areas, including the upper back, shoulders, extensor arms, neck, and upper torso, while the face is often spared. The skin lesions have an annular configuration with raised erythematous borders and central clearing or can be papulosquamous with an eczematous or psoriasiform appearance. A strong association exists with anti-Ro (SS-A) autoantibodies, with a $>80 \%$ of positivity.

Therapy with sunscreens, topical corticosteroids, and antimalarial agents are effective. The lesions heal without scarring or atrophy (as opposed to discoid lupus erythematosus) but may result in prominent dyspigmentation. Severe systemic disease is unusual.

3. $\quad 3.1$ Fixed drug eruption

3.2 History of treatment with drugs

3.3 Avoid the culprit drug, topical application of steroids and short course of oral steroids when severe

Adverse reactions to medications are common and often manifest as a cutaneous eruption. Fixed drug eruptions account for as much as $16-21 \%$ of all cutaneous drug eruptions. The initial eruption is often solitary and frequently located on the lip or genitalia. Rarely, the eruption may be intraoral. Other common locations of the initial lesion are the hip, lower back/sacrum, or proximal extremity. Lesions may persist from days to weeks and then fade slowly to residual oval hyperpigmented patches.

Common culprit drugs are - analgesics, muscle relaxants, sedatives, anticonvulsants and antibiotics

4. Annular plaque psoriasis and pustular psoriasis with probable psoriatic arthropathy.

Pustular psoriasis is an uncommon form of psoriasis consisting of widespread pustules on an erythematous background which may commence with the development of pustules on the periphery of previous psoriatic plaques.

Although idiopathic in most, the following factors reportedly trigger an eruption of pustular psoriasis:

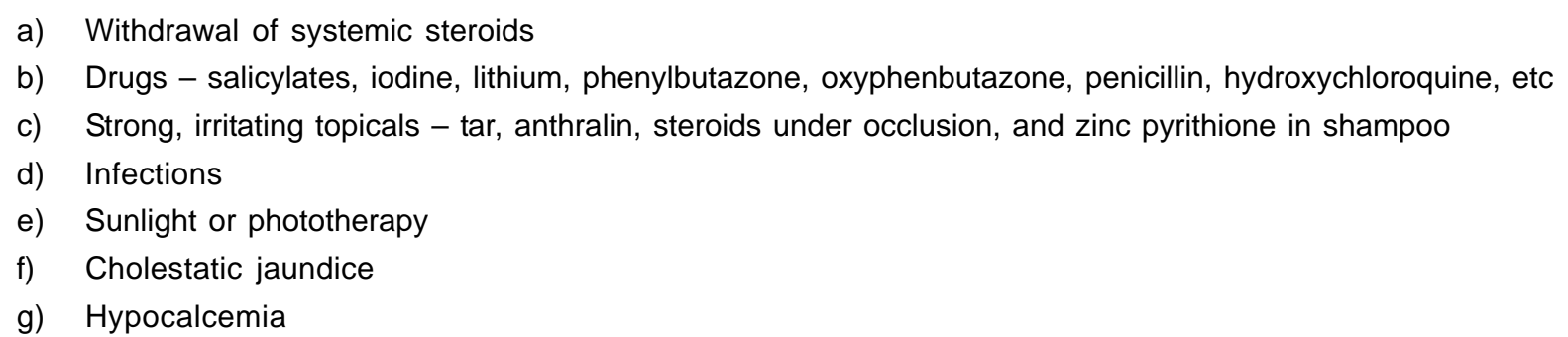

Complications of pustular psoriasis include: secondary bacterial skin infections, hair loss (telogen effluvium), and nail loss, hypoalbuminemia secondary to loss of plasma protein into tissues, hypocalcemia, acute kidney injury, hepatic dysfunction and malnutrition

Oral retinoids, methotrexate, cyclosporine have been used with success.

Compiled by Chalukya Gunasekera Consultant Dermatologist and S A Sathananthan, Senior Registrar in Dermatology, National Hospital of Sri Lanka, Colombo. 